\title{
Development of a porcine model for assessment of mucosal repair following endoscopic resection of the lower gastro- intestinal tract
}

\section{(c) $(>)$}

\author{
Authors \\ Nicolas Etchepare', Jérémy Bregeon², Lucille Quénéhervé1, Sami Haddara', Yann Touchefeu', Michel Neunlist² \\ Emmanuel Coron ${ }^{1,2}$
}

Institutions

1 Institut des Maladies de l'Appareil Digestif, Hôtel Dieu, Centre Hospitalier Universitaire, Nantes, France

2 INSERM U 1235 - TENS, Université de Nantes, Faculté de Médecine, Nantes, France

submitted 23.1.2017

accepted after revision 2.5.2017

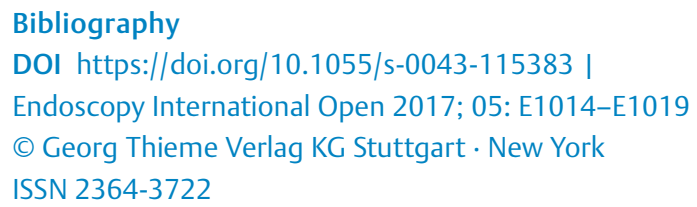

Corresponding author

Emmanuel Coron, Institut des Maladies de l'Appareil

Digestif, CHU de Nantes, 1 place Alexis Ricordeau, 44093

Nantes Cedex 1, France

Fax: +33-2-40083333

emmanuel.coron@chu-nantes.fr

\section{ABSTRACT}

Background and aims Endoscopic mucosal resection (EMR) is widely performed for the treatment of colorectal polyps. However, the pathophysiological mechanisms of mucosal repair, including in situations at high risk of postpolypectomy bleeding, remain largely unknown. The objective of our study was to develop a porcine model of EMR in the lower gastrointestinal tract to monitor mucosal wound healing over time.

Methods Under general anesthesia, five large wounds were created in the lower gastrointestinal tract at different times, i.e. at day $0,3,7,10$, and 14 , by multiband EMR, in each of the six pigs in the study. A colorectal resection was performed at day 14 and the animal euthanized. Repeated endoscopic and endomicroscopic examination, and histological analysis were performed.

Results No complications occurred and all animals reached the study end point. The endoscopic aspect of wound healing evolved into different phases with first a fibrin deposit covering the wounds which then gave way to granulomatous tissue. The size of the wound regressed significantly as early as day 3. Re-epithelialization of the wound started from day 7, and neo-mucosal crypts appeared from day 10. The endomicroscopic analysis described a 'ground glass appearance' from day 3 and irregular crypts from day 10 , which was consistent with histological data. Good agreement between macroscopic, endomicroscopic, and histological parameters of mucosal wound healing was observed in vivo.

Conclusion This study demonstrates for the first time the feasibility of an experimental in vivo porcine model of lower gastrointestinal endoscopic resections to monitor tissue repair. This model might be helpful to document pharmacological approaches for preventing complications of endoscopic procedures performed in humans.

\section{Introduction}

Advances in endoscopic techniques such as either endoscopic mucosal resection (EMR) or endoscopic submucosal dissection allow minimally invasive resections of very large lesions in the colon or rectum [1,2]. Defects of wound healing can account for post-polypectomy bleeding, which still represents the most common complication of endoscopic resections [3]. To prevent delayed bleeding, clip closure after large colorectal defects is an efficient option in most situations [4]. However, this strategy is limited by several factors including anticoagulation or anti-platelet therapies and its cost-efficacy remains to be determined in 'real-life' conditions [5,6]. Beyond prophylactic clip closure, no pharmacological approach has been developed in the lower intestinal tract aimed at enhancing mucosal repair. However, before conducting clinical studies, a better understanding of wound healing mechanisms and/or preclinical assessment of future therapies should be gained through clinically relevant animal models. 


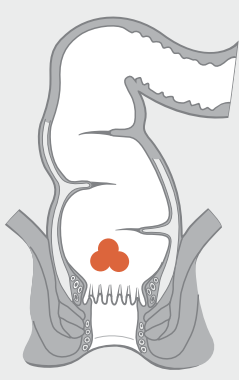

EMR

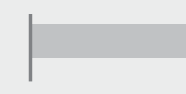

PRD 0

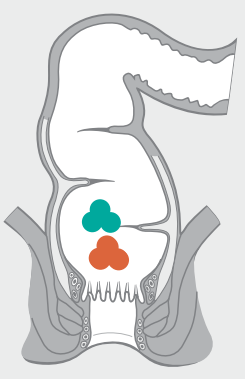

EMR

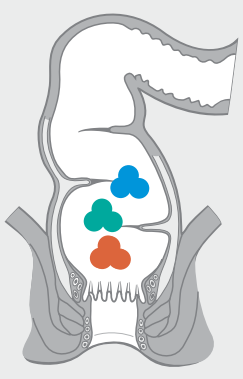

EMR
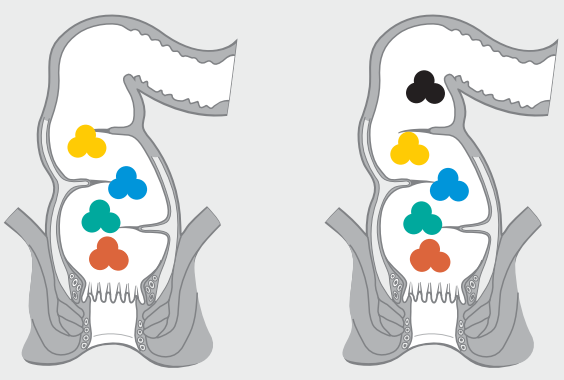

EMR

In vivo analysis

Ex vivo analysis

Fig. 1 Study design. PRD, post-resection day.

Live porcine models have been increasingly used over the last decade $[7,8]$, but most studies focused on simple and short-term outcome parameters such as technical success of the procedure or occurrence of complications or death. However, no analysis of mucosal repair combining in vivo microscopy and standard histology has been performed. In this context, we have developed pig models allowing the monitoring of gastric mucosal repair [9] or intestinal mucosal repair [10] after chemical injuries, using endoscopy and endomicroscopy in addition to standard histology and molecular characterization.

Based upon this expertise, the aim of this study was to develop and assess a porcine model of large EMR in the lower gastrointestinal tract allowing the monitoring of mucosal wound healing over time.

\section{Methods}

\section{Animals}

Six pigs (Sus scrofa domestica) $(35-40 \mathrm{~kg})$ were used. The protocol was approved by the French ethics committee in animal experimentation (Agreement $n^{\circ} 2015.24$ ). General anesthesia was induced by intramuscular administration of $15 \mathrm{mg} / \mathrm{kg}$ of Zolazepamine and the animals were maintained under mechanical ventilation. At the end of the protocol, the animals were euthanized.

\section{EMR procedure}

Immediately before each endoscopy, a rectal enema with physiological serum $(500 \mathrm{~mL}$ of $0.9 \% \mathrm{NaCl}$ ) was performed to allow bowel cleansing.

For each animal, five wounds were created in the lower gastrointestinal tract at different times, i. e. at day $0,3,7,10$, and 14. Each wound consisted of three contiguous resections, performed using an EMR device (Multi-Band Ligation device ${ }^{\mathrm{TM}}$, Cook Medical) under video-endoscopy (EG450, Fujifilm, Japan). EMR was performed after submucosal injection of a mixture of saline and indigo carmine without epinephrine. The first wound was performed $2 \mathrm{~cm}$ above the anal canal. The study design is summarized in $\mathbf{F i g .} \mathbf{1}$.

\section{In vivo examination using endoscopy and endomicroscopy}

Each resection was video-recorded to analyze the wound morphology over time. Endoscopic analysis was performed at day 3 , 7,10 , and 14 . For each wound, the maximal size was measured in the longitudinal axis using a plastic ruler introduced alongside the endoscope. Speed of healing was calculated by measuring each wound at different times during follow-up examinations, and determining the value of the slope in GraphPad Prism software using linear regression analysis.

Probe-based confocal laser endomicroscopy (pCLE) examination (Ultramini-O, CellVizioLab ${ }^{\mathrm{TM}}$, Mauna Kea Technologies) was performed after intravenous injection of $25 \mathrm{~mL}$ of sodium fluorescein solution at $1 \%$ concentration. Three different predetermined areas were examined: (1) the mucosa in the periphery of the wound (i.e. approximately $1 \mathrm{~cm}$ distant from the wound), (2) the border between the wound and the surrounding mucosa, and (3) the center of the wound.

\section{Histological analysis}

After euthanasia, a colorectal resection was performed following laparotomy. The rectum was incised, opened, and flattened. Then each wound site was photographed and measured. Next, each wound was excised and fixed in $4 \%$ formalin before inclusion in paraffin blocks and sectioning $(4 \mu \mathrm{m})$. Sections were stained with hematoxylin-erythrosin-saffron (HES) and scanned for anatomo-pathological analysis (NDP.view software, Hamamatsu). 

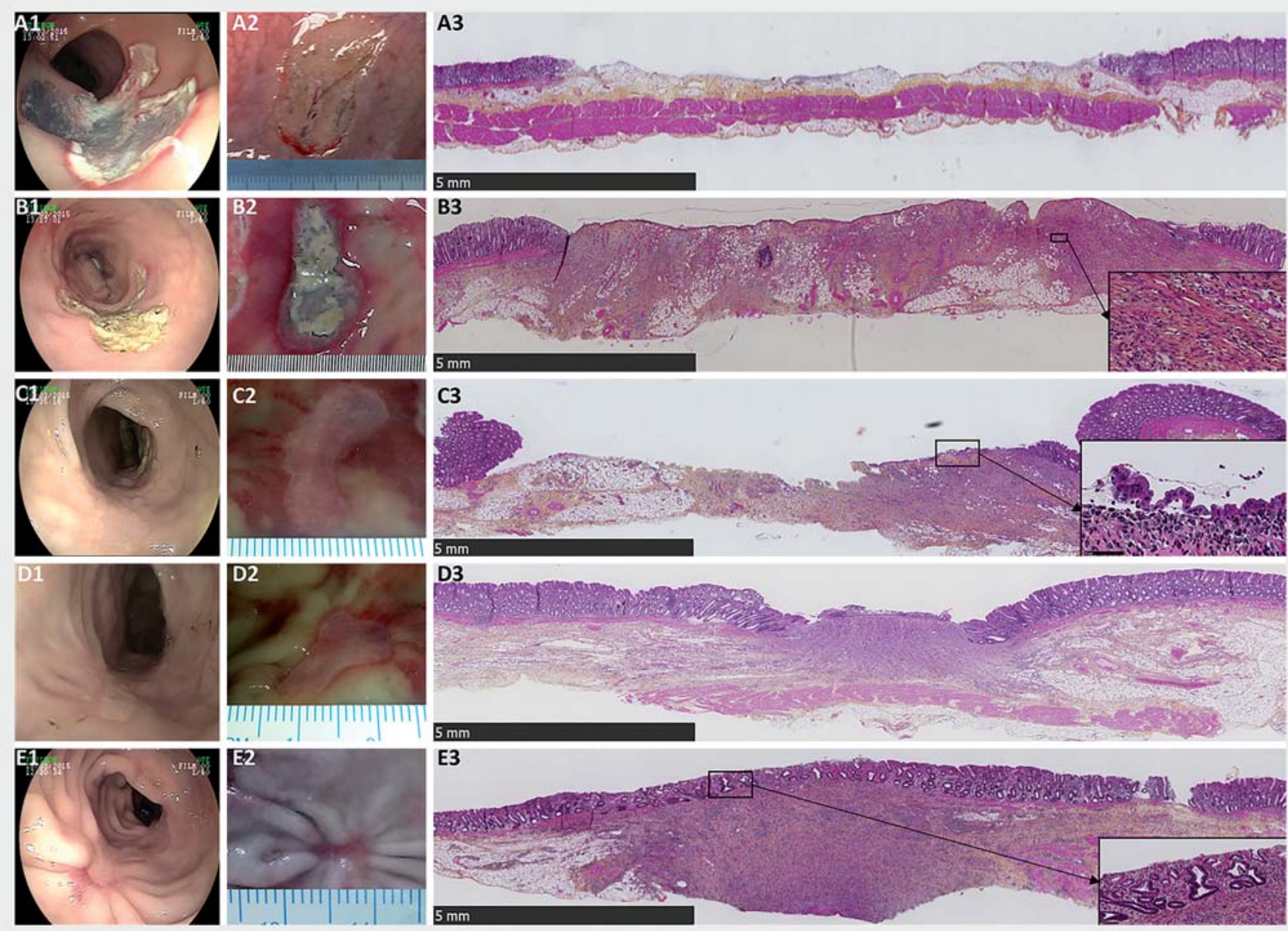

Fig. 2 Evolution of endoscopic, macroscopic, and histologic features at the EMR site. A Appearance immediately after EMR. B Appearance 3 to 4 days after EMR. C Appearance 7 days after EMR. D Appearance 10 days after EMR. E Appearance 14 days after EMR. Submucosa visible immediately after resection $(\mathbf{A} 1,2)$ was recovered by a fibrin-like coat $(\mathbf{B} 1,2)$ then by tissue with granular appearance $(\mathbf{C 1}, \mathbf{2} ; \mathbf{D} 1, \mathbf{2}$; E1, 2). Retracted folds were clearly visible 14 days after resection $(\mathbf{E} 1,2)$. Mucosa and part of the submucosa were removed by EMR (A3). Submucosa was invaded by numerous inflammatory cells (B3, inset, magnified). A monolayer of epithelial cells appeared at the border of the wound, directly on the submucosa (C3, inset, magnified). Epithelial tissue progressively covered the entire wounds giving a structure of epithelial crypts (D3, E3, inset, magnified). For technical reasons, the muscularis propria has been removed from several specimens prior to inclusion in paraffin.

\section{Statistical analysis}

Prism 5.0 (GraphPad Prism 5, La Jolla, CA, United States) software was used for statistical analysis. Data are reported as means \pm standard deviation. Linear regressions and Mann-Whitney $U$ test were performed. A $P$ value $<0.05$ was considered to be statistically significant.

\section{Results}

All pigs $(n=6)$ included in the study had five successful endoscopic procedures. No complications occurred during the endoscopic procedure or during the clinical follow-up.

\section{Endoscopic morphology and size of post-EMR wounds}

Immediately after EMR ( $\triangleright$ Fig. 2, A1), and for all wounds $(n=30)$, a fine layer of submucosa could be identified at the EMR site, containing visible vessels and without exposure of the muscularis propria. On post-resection day (PRD) 3 or 4 ( Fig. 2, B1), $100 \%$ of wounds $(n=24)$ were recovered by a fibrin-like coat and the mucosa surrounding the wound was enanthematous. On PRD 7 ( Fig. 2, C1), the fibrin-like coat took on a granular appearance in all wounds $(n=18)$. On PRD 10, and 14 ( $\triangleright$ Fig. 2, D1 and E1), this granular appearance was unchanged. In addition, retracted folds appeared in the surrounding mucosa, converging to the wound.

Next, we quantified the morphological evolution of the wound ( $>$ Fig.3a). Immediately after EMR, the longitudinal diameter of the wound was $22.3 \pm 4.5 \mathrm{~mm}(\mathrm{n}=28)$. The longitudinal diameter was significantly reduced compared to its initial 


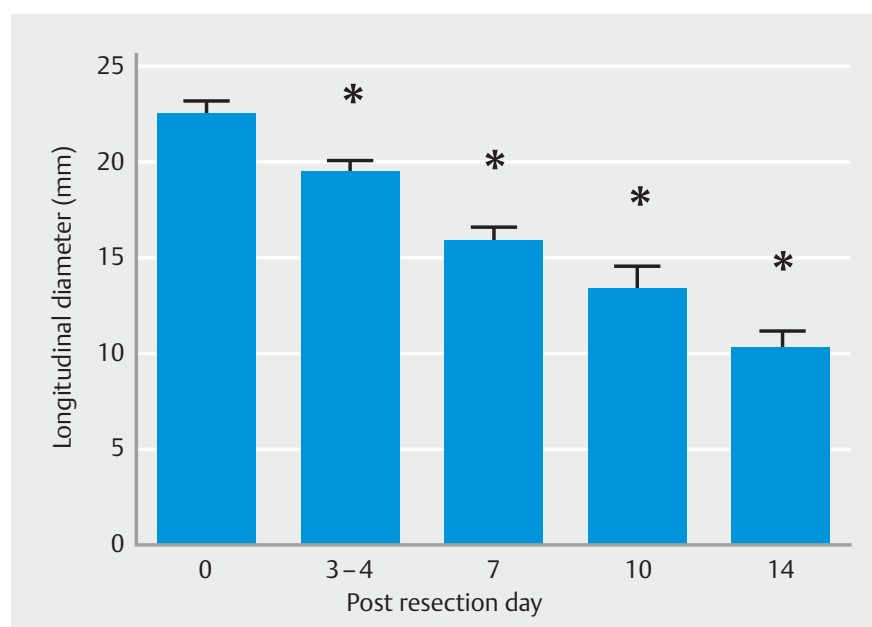

a

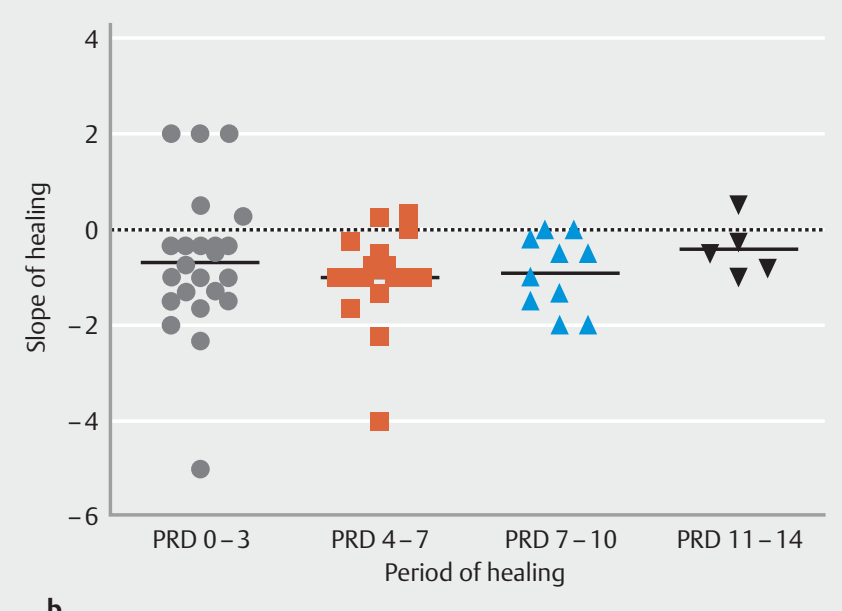

- Fig.3 Quantification of tissue repair parameters following EMR. a Evolution of longitudinal diameter of post-EMR wounds with time. b Evolution of the rate of healing according to the period of healing. ( ${ }^{*} P<0.05$ compared to post-resection day (PRD) 0$)$.

value as early as PRD $3-4(19.3 \pm 3.1 \mathrm{~mm} ; \mathrm{n}=23 ; P=0.012)$. At day 14 , mean size was $10.2 \pm 2.2 \mathrm{~mm}(\mathrm{n}=5, P<0.001)$. In addition, there was no difference in the rate of healing with time ( Fig.3b).

\section{Histological examination}

EMR removed mucosa, muscularis mucosae, and part of the

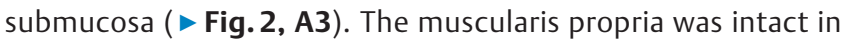
all patients. On PRD 4 ( $\triangleright$ Fig. 2, B3), no epithelial or muscularis mucosae regeneration was observed. Numerous inflammatory cells, mainly neutrophils, were present in the submucosa. On PRD 7 ( Fig. 2, C3), a monolayer of regenerated epithelial cells was present at the border of the wound; no cryptic structure was visible. Moreover, these epithelial cells were located immediately on the remaining inflammatory submucosa without regeneration of muscularis mucosae. On PRD 11 and PRD 14 ( $\triangleright$ Fig.2, D3 and E3), regenerated epithelial cells migrated from the border to the center of the wound and irregular crypts appeared without restoration of the underlying muscularis mucosae. Granulomatous tissue replaced the submucosa. Angiogenesis was present, represented by numerous vessels and the number of inflammatory cells decreased.

\section{pCLE description}

Using PCLE on three predetermined sites, we observed the following aspects ( $>$ Fig. 4): (1) the mucosa in the periphery of the wound was normal with regular crypt shape and distribution. Round crypt lumens as well as regular epithelial lining were clearly identified. This appearance was not modified over time. (2) The border between the post-EMR wound and the surrounding mucosa was easily recognizable without significant changes over time. (3) Targeting the center of the post-EMR wound with pCLE, no structure was initially visible. On PRD 3-4, multiple round cells appeared resulting in a 'ground-glass appearance' without any visible crypts. On PRD 10 -11, a few small, irregular and not very well delimited crypts appeared. The reproducibility of pCLE appearance was good ( $\triangleright$ Table 1 ).

\section{Discussion}

To our knowledge, this is the first report to monitor large wounds after piecemeal EMR over a prolonged period of time. Such an approach has seldom been used in the lower gastrointestinal tract, while several preclinical models of endoscopic wounds have been described in the upper gastrointestinal tract $[11,12]$. Our study also shows the good reproducibility of the model, which could be useful to assess tissue repair and identify novel therapeutic options.

In this study, we described post-EMR mucosal healing in a large animal model. During the first 3 days, the wound was entirely covered by a white fibrin coat, which was then replaced by granulomatous tissue and associated with tissue retraction leading to a reduction in wound size. Histological analysis showed the presence of inflammatory processes in the mucosal defect, the absence of regeneration of muscularis mucosae, and the expansion of the epithelium from the margins to the center of the wound, starting 1 week following EMR. We also correlated ex vivo histological data with in vivo microscopy, performed using pCLE. pCLE has already been used to analyze colonic scars [13] but only during surveillance endoscopies performed at 3-6 months.

Considering the lower gastrointestinal tract, assessment of mucosal repair requires the development of large animal models. Indeed, while healing processes have been studied extensively in the skin in humans [14], data are scarce with regard to the gastrointestinal tract. To our knowledge, this is the first animal model reporting on lower gastrointestinal mucosal repair following endoscopic resections. The understanding of tissue repair mechanisms with time requires several endoscopic and histological examinations, which seems difficult to conceive in humans for ethical reasons. Pigs have important anatomical and physiological similarities to humans, especially considering the gastrointestinal tract [15], and therefore represent an optimal tool for translational research [16]. However, since most models are developed in normal pigs, and due to both species 


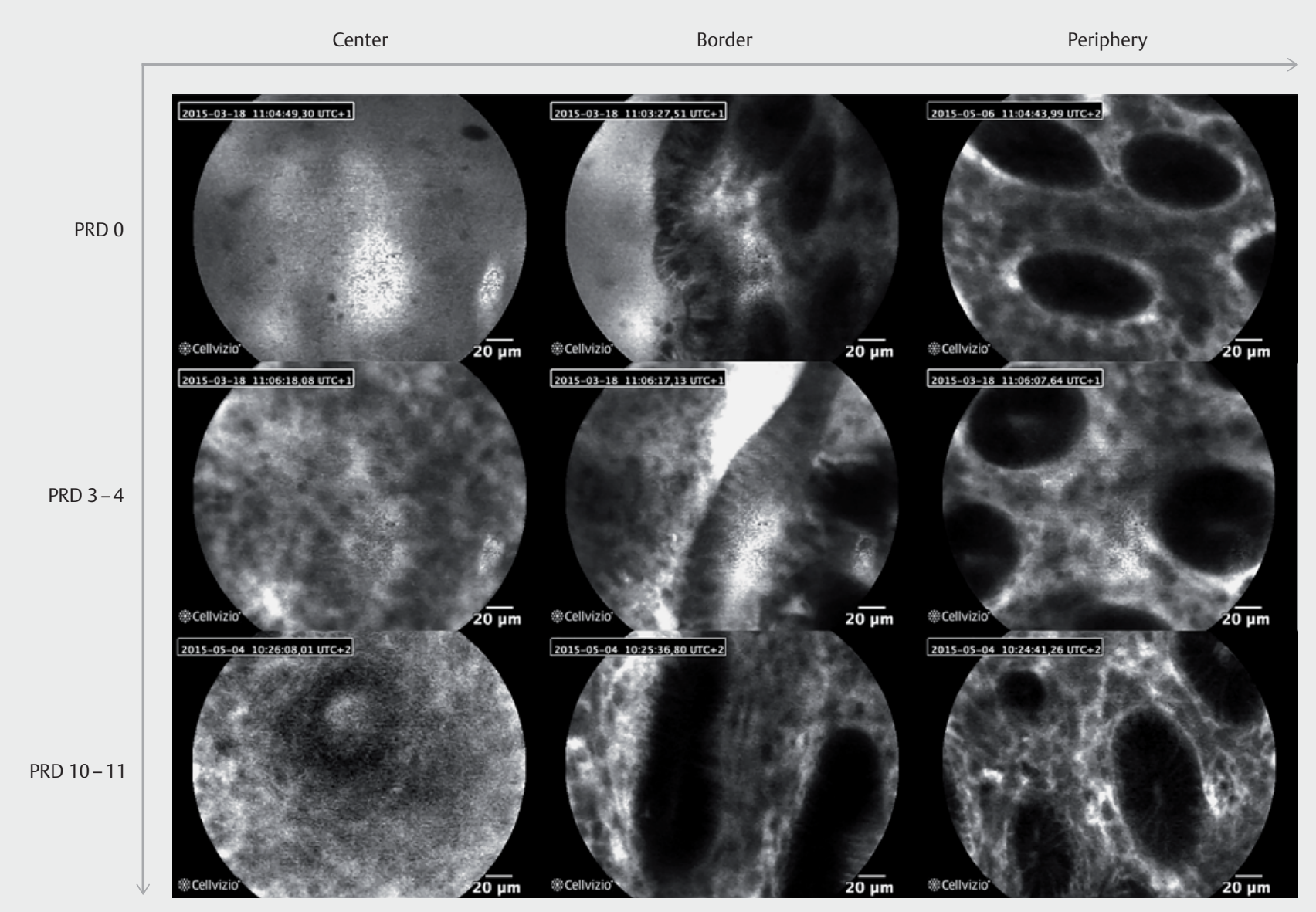

- Fig. 4 pCLE imaging of the EMR site with time. In the center of the post-EMR wound, no structure was visible on post-resection day 0 (PRD 0 ). In contrast, a "ground glass appearance" was visible on PRD 3-4 and some irregular crypts appeared on PRD 10-11. The border of the wound was easily recognizable without modification over time. The peripheral mucosa had a normal appearance with regular crypts at all times of examination.

- Table 1 Summary of pCLE appearance over time.

\begin{tabular}{|l|c|c|c|c|}
\hline Appearance & PRD 0 & PRD 3-4 & PRD 7 & PRD 10-11 \\
\hline No structure, \% & 100 & 0 & 0 & 0 \\
\hline Ground glass, \% & 0 & 100 & 100 & 100 \\
\hline Crypts, \% & 0 & 0 & 0 & 83 \\
\hline
\end{tabular}

barriers and disease specificities, data extrapolated from these models should be considered carefully.

Our study has several limitations. As a "proof of concept" study, it was performed on a limited sample of animals. Future studies will require more animals to validate our findings. Also, we sacrificed the animals on day 14 , which prevented the study of the whole wound healing phase. However, since most delayed complications occur in the first 2 weeks, this time frame is probably sufficient to assess the effects of future wound healing agents.
While demographic and technical factors will lead to more endoscopic resections because of increasing prevalence of colorectal lesions due to demographic and technological evolutions allowing widespread screening and resection devices [1 3], this work might offer broad perspectives. Therefore, we believe that this model has a very high potential for the study of future pharmacological or interventional approaches aimed at promoting tissue repair and preventing complications of endoscopic procedures performed in humans. Among them, butyrate, a short chain fatty acid, is known to promote tissue repair in the case of radiation proctitis or pouchitis, by activating epithelial cell migration and modulating the inflammatory response [17]. The effects of butyrate instillations after mucosal resection in the lower gastrointestinal tract have to be determined since it could promote tissue repair and then reduce delayed complications. Another therapeutic agent might be a self-assembling peptide forming a gel to protect the mucosal defect, as recently proposed by Pioche et al. in the upper gastrointestinal tract [18].

In conclusion, we report for the first time a novel experimental in vivo porcine model of lower gastrointestinal endoscopic resections to monitor tissue repair. We believe that this stand- 
ardized model might be helpful in the future to study drug or device developments aimed at enhancing healing processes in humans.

\section{Acknowledgments}

NE contributed to study design and performed the endoscopy procedures, image analysis, tissue preparation, and data analysis. JB performed data/statistical analyses. LQ, SH and YT contributed to manuscript revision. MN contributed to study design, image and data analysis, and manuscript preparation. EC, principal investigator, was involved in all phases of the work, including initial study design, data analysis and interpretation, and manuscript preparation.

The authors acknowledge Stéphanie Bernardet, David Minault, and Jérémy Hervouet for their technical support at the INSERM Unit 1064 (Laboratoire des Grands Animaux).

\section{Competing interests}

The authors thank Cook Medical ${ }^{\circledR}$ company for its logistical support throughout the study by graciously providing the Multi-Band Ligator. However, the company and its employees had no role in collecting data or writing, correcting, or editing of the manuscript. Emmanuel Coron has been a consultant for Mauna Kea Technologies from 2011 to 2015. The other authors declare no other conflicts of interest.

\section{References}

[1] Zauber AG, Winawer S], O’Brien M] et al. Colonoscopic polypectomy and long-term prevention of colorectal-cancer deaths. NEJM 2012; 23: $687-696$

[2] Fyock C], Draganov PV. Colonoscopic polypectomy and associated techniques. World J Gastroenterol 2010; 16: 3630 -3637

[3] Zhang Q, An S, Chen Z et al. Assessment of risk factors for delayed colonic post-polypectomy hemorrhage: a study of 15553 polypectomies from 2005 to 2013. PloS One 2014; 9: e108290

[4] Zhang Q-S, Han B, Xu J-H et al. Clip closure of defect after endoscopic resection in patients with larger colorectal tumors decreased the adverse events. Gastrointest Endosc 2015; 82: 904 - 909
[5] Kim HS, Kim TI, Kim WH et al. Risk factors for immediate postpolypectomy bleeding of the colon: a multicenter study. Am J Gastroenterol 2006; 101: $1333-1341$

[6] Parikh ND, Zanocco K, Keswani RN et al. A cost-efficacy decision analysis of prophylactic clip placement after endoscopic removal of large polyps. Clin Gastroenterol Hepatol 2013; 11: 1319-1324

[7] Gonzalez LM, Moeser A], Blikslager AT. Porcine models of digestive disease: the future of large animal translational research. Transl Res J Lab Clin Med 2015; 166: 12-27

[8] Rieder E, Mesteri I, Bolton J et al. Endoluminal full-thickness colonwall resection using an over-the-scope-clip: three techniques compared in a porcine survival study. Endoscopy 2012; 44: A14

[9] Coron E, Dewitte M, Aubert P et al. Reversibility of gastric mucosal lesions induced by sodium phosphate tablets and characterized by probe-based confocal laser endomicroscopy. Endosc Int Open 2015; 3: E69-E75

[10] Brégeon J, Coron E, Da Silva ACC et al. Sacral nerve stimulation enhances early intestinal mucosal repair following mucosal injury in a pig model. J Physiol 2016; 94: 4309-4323

[11] Bures J, Kopácová M, Kvetina J et al. Different solutions used for submucosal injection influenced early healing of gastric endoscopic mucosal resection in a preclinical study in experimental pigs. Surg Endosc 2009; 23: $2094-2101$

[12] Honda M, Nakamura T, Hori Y et al. Process of healing of mucosal defects in the esophagus after endoscopic mucosal resection: histological evaluation in a dog model. Endoscopy 2010; 42: 1092-1095

[13] Shahid MW, Buchner AM, Coron E et al. Diagnostic accuracy of probebased confocal laser endomicroscopy in detecting residual colorectal neoplasia after EMR: a prospective study. Gastrointest Endosc 2012; 75: $525-533$

[14] Reinke JM, Sorg H. Wound repair and regeneration. Eur Surg Res 2012; 49: 35-43

[15] Gonzalez LM, Moeser AJ, Blikslager AT. Porcine models of digestive disease: the future of large animal translational research. Transl Res 2015; 166: $12-27$

[16] Moeser AJ, Ryan KA, Nighot PK et al. Gastrointestinal dysfunction induced by early weaning is attenuated by delayed weaning and mast cell blockade in pigs. Am J Physiol Gastrointest Liver Physiol 2007; 293: G413-G421

[17] Hamer HM, Jonkers D, Venema $\mathrm{K}$ et al. Review article: the role of butyrate on colonic function. Aliment Pharmacol Ther 2008; 27: $104-$ 119

[18] Pioche M, Camus M, Rivory J et al. A self-assembling matrix-forming gel can be easily and safely applied to prevent delayed bleeding after endoscopic resections. Endosc Int Open 2016; 4: E415 - E419 